\title{
Additional benefits of GeneXpert MTB/RIF assay for the detection of pulmonary tuberculosis patients with prior exposure to fluoroquinolones
}

This article was published in the following Dove Medical Press journal: Infection and Drug Resistance

\author{
Peijun Tangl,* \\ Ping $\mathrm{Xu}^{1, *}$ \\ Wei Shu ${ }^{2}$ \\ Xiafang Wang' \\ Jian Guo' \\ Huafeng Song' \\ Sumei $\mathrm{Li}^{1}$ \\ Yu Pang ${ }^{2}$ \\ Meiying $\mathrm{Wu}^{\prime}$ \\ 'Department of Tuberculosis, The Fifth \\ People's Hospital of Suzhou, Suzhou, \\ China; ${ }^{2}$ National Clinical Laboratory \\ on Tuberculosis, Beijing Chest \\ Hospital, Capital Medical University, \\ Beijing Tuberculosis and Thoracic \\ Tumor Institute, Beijing, China \\ *These authors contributed equally to \\ this work
}

Correspondence: Yu Pang

Beijing Chest Hospital, Capital Medical

University, Beijing Tuberculosis and

Thoracic Tumor Institute, No. 97,

Machang, Tongzhou District, Beijing,

10I149, China

Tel +86 I0 89509359

Fax +86 10 89509359

Email pangyupound@163.com

Meiying Wu

The Fifth People's Hospital of Suzhou,

No. 10, Changqian Road, Xiangcheng

District, Suzhou, 215100, China

$\mathrm{Tel}+8651265180352$

Fax +8651265I8 0352

Email wu_my@।26.com
Background: We performed a prospective study to investigate the association between prediagnosis exposure to fluoroquinolone (FQ) and laboratory testing results among tuberculosis (TB) patients.

Patients and methods: Each TB-suspected patient provided sputum specimen for mycobacteria growth indicator tube (MGIT) culture and GeneXpert among pulmonary TB patients. Confirmed TB patients and clinically diagnosed TB patients were further enrolled in the final analysis.

Results : A total of $661 \mathrm{~TB}$ patients were included in the final analysis. The distribution of rural TB patients in the FQ-exposed group was significantly higher than that of urban TB patients $(P=0.02)$. GeneXpert showed significantly better positive rate than MGIT technology for TB cases with prior FQ exposure (30.6\% for GeneXpert vs $20.1 \%$ for MGIT, $P=0.01$ ). The positive rate of GeneXpert was significantly higher than that of MGIT for $7-13$ days $(P=0.04)$ and $\geq 14$ days FQ exposure $(P=0.01)$ groups, respectively. We also found that the positive rate of MGIT was significantly decreased from $31.5 \%$ for $<7$ days levofloxacin (LFX) exposure group to $9.4 \%$ for $\geq 14$ days LFX exposure group $(P=0.01$ ), whereas the positive rate of MGIT displayed significant decrease after $7-13$ days exposure to moxifloxacin $(P=0.04)$.

Conclusion: In conclusion, our data demonstrate that TB patients prior to sputum collection are prone to yield negative culture results. GeneXpert could bring additional benefits for the detection of pulmonary TB patients with prior exposure to FQs. In addition, the exposure to moxifloxacin affected mycobacterial culture at an earlier stage compared with LFX.

Keywords: tuberculosis, fluoroquinolone exposure, GeneXpert

\section{Introduction}

Tuberculosis (TB) remains the leading cause of death from a curable infectious disease. ${ }^{1,2}$ It is estimated that a total of 10.4 million new TB cases occurred and $\sim 1.7$ million cases died of this disease worldwide in 2016. ${ }^{1}$ China has the world's third largest TB epidemic, accounting for $11 \%$ of global TB incidence. ${ }^{1}$ Although China has experienced dramatic decline in TB prevalence during the past 20 years, ${ }^{3}$ the emergence of drug-resistant TB hampers efforts at TB control in this country. ${ }^{4}$ The inappropriate use of antibiotics is a major driving force behind the epidemic of drug-resistant TB, especially for fluoroquinolones (FQs). ${ }^{5,6}$

FQs demonstrate excellent in vitro and in vivo activity against Mycobacterium tuberculosis (MTB) and have become cornerstone drugs for the treatment of drug-resistant TB. ${ }^{5,7}$ Unlike other anti-TB agents, FQs are also one of the most widely used antibiotics for a broad spectrum of bacterial infections, especially for treating undiagnosed respiratory 
bacterial infections. ${ }^{89}$ As a consequence, the empirical therapy of suspected infection possibly with FQs masks the diagnosis of active TB, thereby leading to delay in initiation of anti-TB treatment and enhanced TB transmission within the community. ${ }^{9}$

Administration of FQs would transiently reduce the bacterial load and impair the vitality of mycobacteria in patients. ${ }^{7,9}$ Decreased vitality of mycobacteria in the specimen will undoubtedly result in the loss of recovery rate by mycobacterial culture. In contrast, nucleic acid amplification tests have no requirement for viability of mycobacteria; prior exposure to FQ would thus cause less impact on the detection of MTB by molecular diagnostics in comparison with conventional culture. In order to test the null hypothesis, we performed a prospective study to investigate the association between pre-diagnosis exposure to FQ and laboratory testing results by mycobacteria growth indicator tube (MGIT) culture and GeneXpert among pulmonary TB patients.

\section{Patients and methods}

\section{Patients}

We performed a prospective observational study among TB suspected patients in the Fifth People's Hospital of Suzhou, China, from January 2017 to December 2017. A TB-suspected patient was defined as someone presenting at the clinic with a cough of 2 weeks or more ${ }^{10}$ Confirmed TB patients and clinically diagnosed TB patients were further enrolled in final analysis. The definition of TB patients followed the guidelines endorsed by WHO. ${ }^{11}$ We interviewed the patients to collect a comprehensive listing of FQ prescriptions before TB diagnosis. In this setting, third-generation FQs, including levofloxacin (LFX; $500 \mathrm{mg} /$ day) and moxifloxacin (MOX; $400 \mathrm{mg} /$ day), are used for the empirical therapy of suspected respiratory infections. For each TB patient who received FQ exposure, we identified nonexposed patients as the control group. We collected data on age, sex, residence, previous history of TB and exposure history to FQ.

\section{Laboratory examination}

Each patient provided sputum specimen for laboratory examination. Direct smears of sputum specimen were examined using Auramine O staining for acid-fast bacilli. ${ }^{12}$ Sputum specimens were then processed with $N$-acetylcysteine-citrate$\mathrm{NaOH}$ for 15 minutes and were neutralized with PBS $(0.067$ $\mathrm{mol} / \mathrm{L}, \mathrm{pH}=7.4$ ), with subsequent centrifugation at $4,000 \times$ $g$ for 15 minutes. The supernatants were discarded, and the sediments were resuspended in $2 \mathrm{~mL}$ PBS. Then, $0.5 \mathrm{~mL}$ of suspended sputum sediment was used to inoculate one MGIT tube. ${ }^{10}$
Bacterial colonies were harvested 4-8 weeks later for conventional drug susceptibility testing by proportional method as previously reported. ${ }^{13}$ The concentrations of antiTB drugs tested in this study were as follows: isoniazid, 0.2 $\mu \mathrm{g} / \mathrm{mL}$; rifampin (RIF), $40 \mu \mathrm{g} / \mathrm{mL}$; ethambutol, $2 \mu \mathrm{g} / \mathrm{mL}$; streptomycin, $4 \mu \mathrm{g} / \mathrm{mL}$; amikacin, $30 \mu \mathrm{g} / \mathrm{mL}$; and LFX, $2 \mu \mathrm{g} /$ $\mathrm{mL}$. The strain was declared resistant to the specific drug if the growth rate was $>1 \%$ compared to the control. ${ }^{14}$

GeneXpert testing (Cepheid, Sunnyvale, CA, USA) was performed following the manufacturer's instructions. Briefly, sample reagent was added to sputum specimen at a ratio of 2:1. The decontaminated sample was vortexed vigorously twice during 15 minutes of incubation at room temperature. Then, $2 \mathrm{~mL}$ of the mixture was transferred to the test cartridge and loaded into GeneXpert platform. The results were automatically interpreted by the GeneXpert system after 90 minutes.

\section{Statistical analysis}

Statistical analyses were performed using the SPSS software package, version 20.0 (IBM Corporation, Armonk, NY, USA). Chi-squared test or Fisher's exact test was used to compare categorical variables. A $P$-value $<0.05$ was considered statistically significant.

\section{Ethics statement}

This study was approved by the Ethics Committee of the Fifth People's Hospital of Suzhou, and was conducted in accordance with the Declaration of Helsinki. Written informed consent was obtained from each patient enrolled in this study.

\section{Results \\ Patients}

In total, 951 patients with suspected TB were consecutively enrolled in this analysis. By reviewing the laboratory examinations, demographic and clinical data, 290 patients were excluded due to non-TB ( $n=174)$, lack of exposure data to FQ $(n=86)$, contaminated culture results $(n=21)$ or invalid GeneXpert results $(n=9)$. Of 661 TB patients included in the final analysis, 432 patients (65.4\%) experienced no FQ exposure during the course of disease, whereas the other 229 (34.6\%) had prior exposure to FQ, $163(71.2 \%)$ and 66 (28.8\%) of whom were exposed to LFX and MOX, respectively (Figure 1).

\section{Factors associated with FQ exposure}

We first compared the distribution of demographic characteristics and in vitro susceptibility between FQ exposed and nonexposed groups. As shown in Table 1, the TB patients 


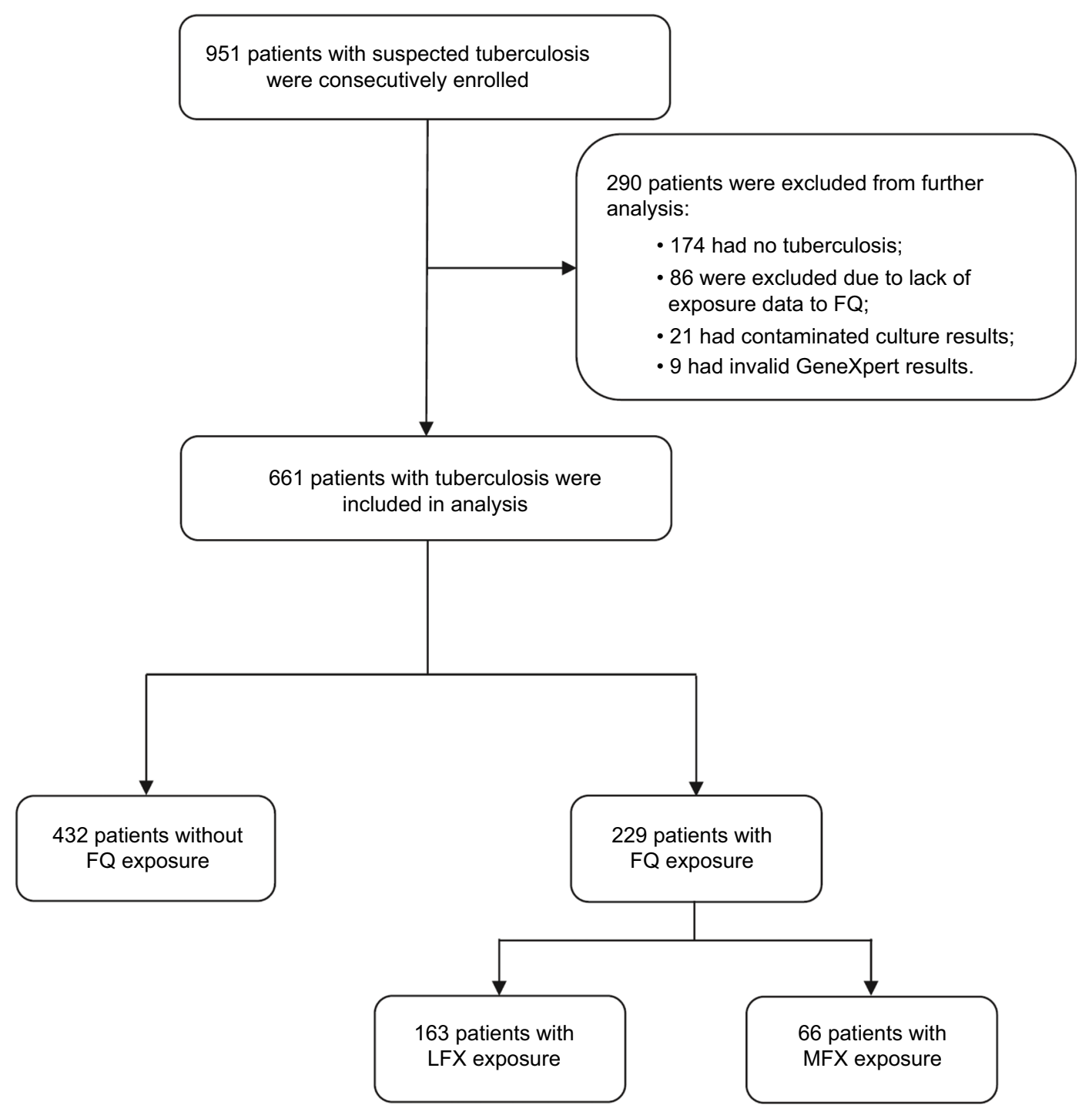

Figure I Enrollment of patients in this study.

Abbreviations: FQ, fluoroquinolone; LFX, levofloxacin; MOX, moxifloxacin.

aged 45-64 years (OR [95\% CI]: 1.80 [1.21-2.67], $P=0.01$ ) and $\geq 65$ years (OR $[95 \% \mathrm{CI}]: 1.73[1.07-2.80], P=0.03$ ) were more likely to be exposed to FQ compared with those aged 25-44 years. In addition, we observed that the distribution of rural TB patients in the FQ-exposed group was significantly higher than that of urban TB patients (OR [95\% CI]: 1.49 [1.08-2.06], $P=0.02$ ). In contrast, no statistical difference was found in the distribution of FQ-exposed patients in gender and drug susceptibility category $(P>0.05)$.

\section{Effect of FQ exposure on detection of MTB by liquid culture and GeneXpert}

One hundred and fifty-four (35.6\%) of 432 TB patients without FQ exposure were detected by the MGIT 960 test, which was similar to that $(34.3 \%, 148 / 432)$ by the GeneXpert test
$(P>0.05)$. For TB cases with prior FQ exposure, the positive rates of MGIT and GeneXpert for detection of MTB were $20.1 \%(46 / 229)$ and 30.6\% (70/229), respectively. The statistical analysis revealed that GeneXpert showed significantly better positive rate than MGIT technology for these cases $(P=0.01)$.

We further analyzed the positive rate according to different FQ exposure duration. As shown in Figure 2, the duration of FQ exposure had no significant effect on the positive rate between MGIT $(31.7 \%, 26 / 82)$ and GeneXpert $(36.6 \%, 30 / 82)$ for the patients with $<7$ days of FQ exposure $(P>0.05)$. On the contrary, the positive rate of GeneXpert was significantly higher than that of MGIT for $7-13$ days $(17.4 \%$ for MGIT vs $30.2 \%$ for GeneXpert, $P=0.04$ ) and $\geq 14$ days ( $8.2 \%$ for MGIT vs $23.0 \%$ for GeneXpert, $P=0.03$ ) groups, respectively. 
Table I Demographic and clinical characteristics of TB patients enrolled in this study

\begin{tabular}{|c|c|c|c|c|c|}
\hline \multirow[t]{2}{*}{ Characteristics } & \multicolumn{3}{|l|}{ No. of patients (\%) } & \multirow[t]{2}{*}{ ORs $(95 \% \mathrm{Cl})$} & \multirow[t]{2}{*}{$P$-value } \\
\hline & $\begin{array}{l}\text { FQ-exposed group } \\
(n=229)\end{array}$ & $\begin{array}{l}\text { FQ-nonexposed group } \\
(n=432)\end{array}$ & $\begin{array}{l}\text { Total } \\
\text { n (\%) }\end{array}$ & & \\
\hline \multicolumn{6}{|l|}{ Age (years) } \\
\hline$<25$ & $32(14.0)$ & $87(20.1)$ & $119(18.0)$ & $0.87(0.54-\mid .4 \mathrm{I})$ & 0.57 \\
\hline $25-44$ & $80(34.9)$ & $189(43.8)$ & $269(40.7)$ & Ref. & - \\
\hline $45-64$ & $76(33.2)$ & $100(23.1)$ & $176(26.6)$ & $1.80(1.2 \mathrm{I}-2.67)$ & 0.01 \\
\hline$\geq 65$ & $41(17.9)$ & $56(13.0)$ & 97 (I4.7) & $1.73(1.07-2.80)$ & 0.03 \\
\hline \multicolumn{6}{|l|}{ Gender } \\
\hline Male & $160(69.6)$ & $274(63.4)$ & $434(65.7)$ & $1.34(0.95-1.89)$ & 0.10 \\
\hline Female & $69(30.1)$ & $158(36.6)$ & $227(34.1)$ & Ref. & - \\
\hline \multicolumn{6}{|l|}{ Residence } \\
\hline Rural & $94(41.0)$ & $220(50.9)$ & $314(47.5)$ & Ref. & - \\
\hline Urban & $135(59.0)$ & $212(49.1)$ & $347(52.5)$ & $1.49(1.08-2.06)$ & 0.02 \\
\hline \multicolumn{6}{|l|}{ Resistance to: $^{a}$} \\
\hline RIF & $3(6.5)$ & II (7.I) & $14(7.0)$ & $0.91(0.24-3.40)$ & 1.00 \\
\hline $\mathrm{INH}$ & $5(10.9)$ & $18(11.7)$ & $23(11.5)$ & $0.92(0.32-2.64)$ & 0.88 \\
\hline SM & $7(15.2)$ & $25(16.2)$ & $32(16.0)$ & $0.93(0.37-2.30)$ & 0.87 \\
\hline EMB & $2(4.3)$ & $5(3.2)$ & $7(3.5)$ & $1.36(0.25-7.22)$ & 0.66 \\
\hline LFX & $2(4.3)$ & $7(4.5)$ & II (4.5) & $0.96(0.19-4.76)$ & 1.00 \\
\hline AMK & $2(4.3)$ & $6(3.9)$ & $8(4.0)$ & $1.12(0.22-5.75)$ & 1.00 \\
\hline
\end{tabular}

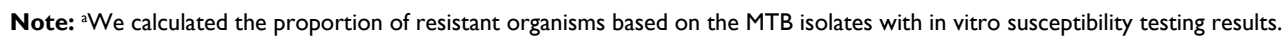

Abbreviations: AMK, amikacin; EMB, ethambutol; FQ, fluoroquinolone; INH, isoniazid; LFX, levofloxacin; RIF, rifampin; SM, streptomycin; TB, tuberculosis.

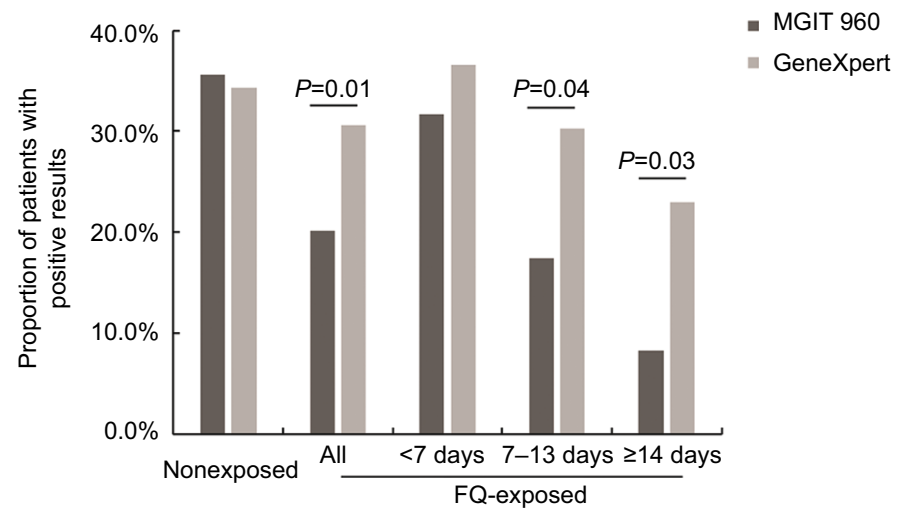

Figure 2 Comparison of detection rates for MTB between GeneXpert and MGIT according to FQ exposure stratification. Abbreviations: FQ, fluoroquinolone; MTB, Mycobacterium tuberculosis; MGIT, mycobacteria growth indicator tube.

\section{Comparison of LFX and MOX exposure on the detection of MTB}

Further analysis was performed to determine whether LFX and MOX exposure produced different impact on the detection of MTB. We found that the positive rate of MGIT was decreased from $31.5 \%$ (17/54) for $<7$ days LFX exposure group to $9.4 \%$ (5/53) for $\geq 14$ days LFX exposure group, and the difference was statistically significant between $<7$ and $\geq 14$ days groups $(P=0.01)$. For patients with prior exposure to MOX, the statistical analysis revealed that the positive rate of MGIT displayed significant decrease after 7-13 days of exposure to MOX $(10.0 \%, 3 / 30)$ compared with that in $<7$ days exposure group $(32.1 \%, 9 / 28 ; P=0.04)$. In contrast, no significant difference was observed in the detection rate of GeneXpert for MTB between these groups $(P>0.05$; Table 2$)$.

\section{Discussion}

The escalating use of FQs for routine bacterial infections is associated with possible masking of active TB. ${ }^{9}, 15$ We examined the impact of FQ use on the detection rates of GeneXpert and MGIT for MTB. Our data demonstrated that TB patients who received $\mathrm{FQ}$ for $\geq 7$ days prior to sputum collection were 
Table 2 Laboratory results by MGIT 960 and GeneXpert among patients with exposure to $\mathrm{FQ}$

\begin{tabular}{|c|c|c|c|c|}
\hline \multirow[t]{2}{*}{ Group } & \multirow[t]{2}{*}{$\begin{array}{l}\text { Laboratory } \\
\text { method }\end{array}$} & \multicolumn{3}{|c|}{$\begin{array}{l}\text { No. of patients with positive } \\
\text { laboratory results (\%) }\end{array}$} \\
\hline & & $<7$ days & $7-13$ days & $\geq 14$ days \\
\hline \multirow[t]{3}{*}{ LFX } & MGIT 960 & $17(3 \mid .5)$ & $13(23.2)$ & $5(9.4)^{*}$ \\
\hline & GeneXpert & $20(37.0)$ & $17(30.4)$ & $13(24.5)$ \\
\hline & Total & $54(100.0)$ & $56(100.0)$ & $53(100.0)$ \\
\hline \multirow[t]{3}{*}{ MOX } & MGIT 960 & $9(32.1)$ & $3(10.0)^{*}$ & $\mathrm{I}(\mathrm{I} 2.5)$ \\
\hline & GeneXpert & $10(35.7)$ & $9(30.0)$ & $\mathrm{I}(12.5)$ \\
\hline & Total & $24(100.0)$ & $30(100.0)$ & $8(100.0)$ \\
\hline
\end{tabular}

Note: ${ }^{\mathrm{T} T h e}$ asterisks represent statistically significant differences from the control (patients without exposure to $\mathrm{FQ}$ ).

Abbreviations: FQ, fluoroquinolone; LFX, levofloxacin; MOX, moxifloxacin; MGIT, mycobacteria growth indicator tube.

more prone to yield negative culture results than nonexposed patients. Similar to our observations, a recent study from South Africa revealed that patients recently exposed to $\geq 5$ days of FQ were less likely to be smear positive, resulting in an increased time to treatment. ${ }^{9}$ In addition, GeneXpert exhibited better sensitivity to detect MTB from those with $\geq 7$ days exposure to $F Q$, suggesting that GeneXpert could bring additional benefits for the diagnosis of TB patients with prior exposure to $\mathrm{FQ}$. This appeared to be mainly because the molecular diagnostics does not depend on the presence of viable organisms. There is no doubt that the tubercle bacillus cells undergo loss of viability when exposed to FQs, and the prolonged duration of initial empirical FQ treatment is associated with lower recovery rate in clinical specimens. However, several previous reports demonstrated that $>50 \%$ of the bacilli observed on smear microscopy after the 2-month intensive phase were not alive, ${ }^{16,17}$ indicating that the dead tubercle bacillus persists in the pulmonary lesion despite appropriate treatment and apparent recovery. As a consequence, GeneXpert MTB/RIF assay could be used as an alternative for the detection of MTB for patients with prior exposure to FQs.

Another interesting finding of this study is that the exposure to MOX affected mycobacterial culture at an earlier stage compared with LFX, reflecting that MOX has superior overall efficacy over LFX against MTB. This hypothesis is confirmed by other in vitro and in vivo experimental results. ${ }^{18-20}$ On one hand, two animal studies have suggested that MOX concentrates inside the macrophages and pulmonary lesions to a greater extent than LFX. ${ }^{19,21}$ Hence, the greater potency of MOX relative to LFX majorly contributed to the improved drug delivery to the sites of infection. On the other hand, FQs exhibit dose- and concentration-dependent killing kinetics against MTB and other pathogens. ${ }^{22-24} \mathrm{~A}$ recent study showed that LFX at $1,000 \mathrm{mg}$ daily yielded a similar early bactericidal activity to a $400 \mathrm{mg}$ daily dose of MOX. ${ }^{19}$ Hence, the reduced $500 \mathrm{mg}$ daily dose of LFX may be less potent than $400 \mathrm{mg}$ daily dose of MOX against MTB in clinical practice, which may be an additional reason for the greater EBA of MOX in our observations.

Previous studies have demonstrated potential detrimental effects of FQ use in patients with undiagnosed TB, including an increased risk of diagnosis delay and mortality. ${ }^{79}$ In this study, rural residence was associated with greater FQ prescription rate prior to $\mathrm{TB}$ diagnosis, which was also an important determinant of patient delay in seeking and receiving care for TB in China. ${ }^{25}$ Despite the implementation of New Cooperative Medical System in rural China since 2003, rural patients have to suffer high out-of-pocket costs on diagnosis and treatment for TB. ${ }^{26,27}$ Therefore, the poor economic status becomes a major barrier to access to health care for patients living in rural areas. In addition, the significant quality deficits among village clinics and township health centers in the management of TB patients have been reported by Sylvia et al, ${ }^{28}$ and the sizable gap between physician knowledge and practice would lead to the incorrect interpretations of clinical symptoms associated with TB, thereby resulting in the empirical treatment with antibiotics. Given that rural areas have consistently higher TB prevalence than urban areas in China, concerns have been raised regarding how to take effective actions to protect the most vulnerable members of the population, particularly the rural poor.

This study has several obvious limitations. First, this study was only carried out in a single hospital. The small sample size of patients with MOX exposure may undermine the reliability of our observation. Second, the patients enrolled in this study had a fixed-dose LFX or MOX. It is difficult to conclude whether increased dose of FQ would accelerate the reduction of viable MTB in pulmonary lesions, thereby affecting the performance of laboratory examination. Third, we also found that $\sim 10 \%$ of TB patients with long-period FQ exposure ( $\geq 14$ days) yielded positive culture results. Unfortunately, the molecular characteristics of $g y r A$ and $\operatorname{gyr} B$ conferring FQ resistance and minimal inhibitory concentrations were not analyzed in our study. ${ }^{5,29}$ Fourth, although GeneXpert could identify additional TB cases with prior exposure to FQ, some TB cases failed to be detected by both diagnostic tests. The major explanation may be due to the fact that the GeneXpert assay targets a single copy gene of rpoB, which may be associated with the decreased sensitivity compared to the multicopy MTB target..$^{30}$ Recently, a new-generation GeneXpert Ultra has been developed by adding additional multicopy amplification targets (IS6110 and IS1081). ${ }^{31}$ The 
application of this novel test will serve as an alternative way that the additional cases could be identified. Fifth, it should be noted that GeneXpert cannot differentiate between live and dead tubercle bacilli, thereby resulting in increasing probability of false-positive cases. Despite these limitations, a number of important clinical implications flow from our findings. On one hand, we present a new laboratory diagnostic diagram for TB suspects with FQ exposure. On the other hand, the high rate of FQ exposure prior to TB diagnosis highlights the urgent need to strengthen the guidance of FQ prescription used for empirical treatment against bacterial infections.

In conclusion, our data demonstrate that $\mathrm{TB}$ patients who received FQ for $\geq 7$ days prior to sputum collection are more prone to yield negative culture results than nonexposed patients. As an alternative, GeneXpert MTB/RIF assay could bring additional benefits for the detection of pulmonary $\mathrm{TB}$ patients with prior exposure to FQs. In addition, the exposure to MOX affected mycobacterial culture at an earlier stage compared with LFX. The high rate of FQ exposure prior to TB diagnosis highlights the urgent need to strengthen the guidance of FQ prescription used for empirical treatment against bacterial infections.

\section{Acknowledgments}

This study was supported by the Jiangsu Youth Medical Talents Project (QNRC2016226), Suzhou Municipal Science and Technology Bureau Science and Technology Project for People's Livelihood (SS201541 and SS201656), Suzhou City Key Clinical Disease Diagnosis and Treatment Technology Special Project (LCZX201514), Yangfan Foundation of Innovation Alliance on Tuberculosis Diagnosis and Treatment (Beijing) and China Tuberculosis Clinical Trial Consortium (2018KYJJ007). We would like to thank staff in the Fifth People's Hospital of Suzhou for their assistance in implementing this work.

\section{Disclosure}

The authors report no conflicts of interest in this work.

\section{References}

1. World Health Organization (WHO). Global tuberculosis report 2017. Geneva: WHO; 2017. Available from: WHO/HTM/TB/2017.23. Accessed September 18, 2018.

2. Pang Y, Du J, Qin ZZ, et al. An overview on tuberculosis-specific hospitals in China in 2009: results of a national survey. Eur Respir J. 2016;47(5):1584-1587.

3. Wang L, Zhang H, Ruan Y, et al. Tuberculosis prevalence in China, 1990-2010; a longitudinal analysis of national survey data. Lancet. 2014;383(9934):2057-2064.

4. Zhao Y, Xu S, Wang L, et al. National survey of drug-resistant tuberculosis in China. N Engl J Med. 2012;366(23):2161-2170.
5. Zhang Z, Lu J, Wang Y, Pang Y, Zhao Y. Prevalence and molecular characterization of fluoroquinolone-resistant Mycobacterium tuberculosis isolates in China. Antimicrob Agents Chemother. 2014;58(1):364-369.

6. Matteelli A, Roggi A, Carvalho AC. Extensively drug-resistant tuberculosis: epidemiology and management. Clin Epidemiol. 2014;6:111-118.

7. Lee JY, Lee HJ, Kim YK, et al. Impact of fluoroquinolone exposure prior to tuberculosis diagnosis on clinical outcomes in immunocompromised patients. Antimicrob Agents Chemother. 2016;60(7):4005-4012.

8. Long R, Chong H, Hoeppner V, et al. Empirical treatment of communityacquired pneumonia and the development of fluoroquinolone-resistant tuberculosis. Clin Infect Dis. 2009;48(10):1354-1360.

9. Jeon CY, Calver AD, Victor TC, Warren RM, Shin SS, Murray MB. Use of fluoroquinolone antibiotics leads to tuberculosis treatment delay in a South African gold mining community. Int J Tuberc Lung Dis. 2011;15(1):77-83.

10. Tan Y, Li Q, Wang Q, et al. Evaluation of the MTBDRplus 2.0 assay for the detection of multidrug resistance among persons with presumptive pulmonary TB in China. Sci Rep. 2017;7(1):3364.

11. World Health Organization (WHO). Definitions and Reporting Framework for Tuberculosis. Geneva: WHO; 2013. Available from: WHO/ HTM/TB/2013.2. Accessed March 1, 2013.

12. Xia H, Song YY, Zhao B, et al. Multicentre evaluation of Ziehl-Neelsen and light-emitting diode fluorescence microscopy in China. Int J Tuberc Lung Dis. 2013;17(1):107-112.

13. Zhang Z, Wang Y, Pang Y, Kam KM, Land GA. Ethambutol resistance as determined by broth dilution method correlates better than sequencing results with embB mutations in multidrug-resistant Mycobacterium tuberculosis isolates. J Clin Microbiol. 2014;52(2):638-641.

14. World Health Organization (WHO). Guidelines for Surveillance of Drug Resistance in Tuberculosis. 5th ed. Geneva: WHO; 2015. Available from: WHO/HTM/TB/2015.13. Accessed June 1, 2015.

15. Chang KC, Leung CC, Yew WW, et al. Newer fluoroquinolones for treating respiratory infection: do they mask tuberculosis? Eur Respir J. 2010;35(3):606-613.

16. Ramarokoto H, Randriamiharisoa H, Rakotoarisaonina A. Bacteriological follow-up of tuberculosis treatment: a comparative study of smear microscopy and culture results at the second month of treatment. Int $J$ Tuberc Lung Dis. 2002;6(10):909-912.

17. Zhao FZ, Levy MH, Wen S. Sputum microscopy results at two and three months predict outcome of tuberculosis treatment. Int J Tuberc Lung Dis. 1997;1(6):570-572.

18. Wang JY, Wang JT, Tsai TH, et al. Adding moxifloxacin is associated with a shorter time to culture conversion in pulmonary tuberculosis. Int J Tuberc Lung Dis. 2010;14(1):65-71.

19. Ahmad Z, Tyagi S, Minkowski A, Peloquin CA, Grosset JH, Nuermberger EL. Contribution of moxifloxacin or levofloxacin in second-line regimens with or without continuation of pyrazinamide in murine tuberculosis. Am J Respir Crit Care Med. 2013;188(1):97-102.

20. Johnson JL, Hadad DJ, Boom WH. Early and extended early bactericidal activity of levofloxacin, gatifloxacin and moxifloxacin in pulmonary tuberculosis. Int J Tuberc Lung Dis. 2006;10(6):605-612.

21. Michot JM, Seral C, Van Bambeke F, Mingeot-Leclercq MP, Tulkens PM. Influence of efflux transporters on the accumulation and efflux of four quinolones (ciprofloxacin, levofloxacin, garenoxacin, and moxifloxacin) in J774 macrophages. Antimicrob Agents Chemother. 2005;49(6):2429-2437.

22. Firsov AA, Vostrov SN, Lubenko IY, Drlica K, Portnoy YA, Zinner SH. In vitro pharmacodynamic evaluation of the mutant selection window hypothesis using four fluoroquinolones against Staphylococcus aureus. Antimicrob Agents Chemother. 2003;47(5):1604-1613.

23. Sirgel FA, Botha FJ, Parkin DP, et al. The early bactericidal activity of ciprofloxacin in patients with pulmonary tuberculosis. Am J Respir Crit Care Med. 1997;156(3 Pt 1):901-905.

24. Yoshimatsu T, Nuermberger E, Tyagi S, Chaisson R, Bishai W, Grosset J. Bactericidal activity of increasing daily and weekly doses of moxifloxacin in murine tuberculosis. Antimicrob Agents Chemother. 2002;46(6):1875-1879. 
25. LiY, Ehiri J, Tang S, et al. Factors associated with patient, and diagnostic delays in Chinese TB patients: a systematic review and meta-analysis. BMC Med. 2013;11(1):156.

26. Ma J, Xu J, Zhang Z, Wang J. New cooperative medical scheme decreased financial burden but expanded the gap of income-related inequity: evidence from three provinces in rural China. Int $J$ Equity Health. 2016;15(1):72.

27. Li J, Shen X, Yeoh EK, Chung PH. Tuberculosis control programs and challenges in developed cities with intermediate disease burden: China experience. J Thorac Dis. 2017;9(5):E525-E528.

28. Sylvia S, Xue H, Zhou C, et al. Tuberculosis detection and the challenges of integrated care in rural China: a cross-sectional standardized patient study. PLoS Med. 2017;14(10):e1002405.
29. Avalos E, Catanzaro D, Catanzaro A, et al. Frequency and geographic distribution of gyrA and gyrB mutations associated with fluoroquinolone resistance in clinical Mycobacterium tuberculosis isolates: a systematic review. PLoS One. 2015;10(3):e0120470.

30. Akkerman OW, van der Werf TS, de Boer M, et al. Comparison of 14 molecular assays for detection of Mycobacterium tuberculosis complex in bronchoalveolar lavage fluid. J Clin Microbiol. 2013;51(11): 3505-3511.

31. Chakravorty S, Simmons AM, Rowneki M, et al. The new Xpert MTB/ RIF ultra: improving detection of Mycobacterium tuberculosis and resistance to rifampin in an assay suitable for point-of-care testing. MBio. 2017;8(4):e00812-17.
Infection and Drug Resistance

\section{Publish your work in this journal}

Infection and Drug Resistance is an international, peer-reviewed openaccess journal that focuses on the optimal treatment of infection (bacterial, fungal and viral) and the development and institution of preventive strategies to minimize the development and spread of resistance. The journal is specifically concerned with the epidemiology of antibiotic

\section{Dovepress}

resistance and the mechanisms of resistance development and diffusion in both hospitals and the community. The manuscript management system is completely online and includes a very quick and fair peerreview system, which is all easy to use. Visit http://www.dovepress.com/ testimonials.php to read real quotes from published authors.

Submit your manuscript here: https://www.dovepress.com/infection-and-drug-resistance-journal 